\title{
Short-term indirect interactions between two moth (Lepidoptera: Noctuidae) species mediated by shared parasitoids: The benefit of being scarce
}

\author{
TIIT TEDER ${ }^{1 *}$ and TOOMAS TAMMARU ${ }^{1,2}$ \\ ${ }^{1}$ Institute of Zoology and Botany, Riia 181, 51014 Tartu, Estonia; e-mail: tiit@zbi.ee \\ ${ }^{2}$ Institute of Zoology and Hydrobiology, Vanemuise 46, University of Tartu, 51014 Tartu, Estonia
}

Key words. Parasitoids, indirect effects, apparent competition, coexistence, host preference, frequency dependence, Ichneumonidae, Noctuidae, Nonagria typhae, Archanara sparganii

\begin{abstract}
Despite the impact of parasitoids on insect populations being extensively studied, indirect parasitoid-mediated effects remain rarely documented in natural communities. We examined the influence of shared parasitoids on the interactions between two functionally monophagous moths, Nonagria typhae and Archanara sparganii. The moths showed a considerable variation in terms of relative abundance and the degree of phenological synchrony between the species. On average, parasitism levels caused by shared parasitoids did not differ between the two host species. Relative parasitism levels of the two hosts, however, varied considerably among different samples. Percentage parasitism of the scarcer species, $A$. sparganii, thus could not be fully explained by that of the dominant species, $N$. typhae. The results indicated that $A$. sparganii may benefit from the presence of $N$. typhae. In particular, both low relative density as well as high phenological synchrony with $N$. typhae reduced parasitism levels in $A$. sparganii. The case thus indicates the presence of parasitoid-mediated indirect effects between the coexisting herbivores. The patterns of host use observed in this study are consistent with the scenario of frequency-dependent host use caused by changes in parasitoid behavior. Such a host use by parasitoids is suggested to promote numerical stability and coexistence of the moth species in the system studied.
\end{abstract}

\section{INTRODUCTION}

An interaction between two species is called indirect when the effects of one species on another are mediated by some third species (Strauss, 1991; Wootton, 1994). Natural-enemy-mediated indirect effects may arise either through functional (short-term effects) or numerical response (long-term, or transgenerational effects) of the enemy to the prey population. Enemy-mediated indirect effects have been suggested to play a significant role in natural communities affecting community structure and population dynamics of the species involved (Jeffries \& Lawton, 1984; Holt \& Lawton, 1994; Müller \& Godfray, 1999). However, the empirical evidence on such effects is still scarce (Bonsall \& Hassell, 1999; Chaneton \& Bonsall, 2000). In a recent review, Chaneton \& Bonsall (2000) found as few as 21 studies addressing questions about, or interpreting results, in the context of apparent competition. The evidence on horizontal, positive indirect effects in communities is even more scattered and comes mainly from herbivore-mediated interactions between plants (Houston et al., 1993; Olofsson et al., 1999).

Interspecific interactions between coexisting herbivorous insects can be mediated both by lower (host plant) and higher (natural enemies) trophic levels. While hostplant-mediated effects are usually mutually negative (resource competition), the type and strength of indirect interactions mediated by shared predators or parasitoids are less obvious. The outcome - are the interactions mutually positive (apparent mutualism), mutually negative (apparent competition), or non-reciprocal (indirect amensalism) - is suggested to depend on various factors, e.g. foraging behavior of the natural enemies, relative feeding habits or competitive abilities of victim species (Jeffries \& Lawton, 1984; Holt, 1987; Holt \& Kotler, 1987; Holt \& Lawton, 1994; Abrams et al., 1998).

In the case of herbivorous insects, parasitoids have probably the greatest potential of mediating indirect effects between coexisting species. The type and strength of parasitoid-mediated indirect interactions are presumably sensitive to the composition of parasitoid community. This is because of a high diversity of lifehistories (e.g. koinobionts vs idiobionts, ectoparasitoids vs endoparasitoids) and diverse behavioral repertoire (host preference, host switching etc.) among parasitoids. Empirical studies on various systems are therefore required to understand how widespread parasitoidmediated indirect effects may be in natural communities, and which factors influence the expression of such effects. While manipulative studies are necessary to reveal causal relationships, an analysis of correlative data would estimate the range and relevance of the effects in the field.

In the present field study, we demonstrate a pattern consistent with indirect, parasitoid-mediated interactions between a pair of insect herbivores exploiting a common host plant. The species studied are functionally monophagous and their parasitoids lack alternative host species in the study areas. In its relative isolation, the system is thus well suited for examining indirect interactions in the field. We document variation in relative parasitism levels of the two host species, and analyze possible factors

\footnotetext{
* Corresponding author. Tel. (+372 7) 473 272; fax: (+372 7) 383013.
} 
responsible for the patterns observed. Attention is paid to the possible role of indirect effects mediated by variations in relative abundance and relative phenological distribution of the herbivores. Finally, we discuss possible consequences of the detected indirect interactions on the population dynamics of the moth species and community structure.

\section{MATERIAL AND METHODS}

\section{Study species}

The study system was based on Typha latifolia L. (Typhaceae), a rhizomatous perennial plant up to $3 \mathrm{~m}$ in height forming dense stands in damp sites. Larvae of four moth species have been found feeding on leaves and stems of $T$. latifolia (Teder et al., 1999; Teder \& Tammaru, 2002). This study was, however, restricted to the two most abundant species, Nonagria typhae Thunberg and Archanara sparganii Esper (Lepidoptera: Noctuidae). The proportion of other species remained $<1 \%$ of all sampled larvae. The species studied are close taxonomically and have a high resemblance in feeding biology and phenology (pers. obs.). They overwinter as eggs that hatch in spring. At the beginning of the season larvae feed on the aerial roots of the host plant, while later they switch to endophytic feeding in the shoots (Galichet et al., 1992). One larva usually feeds on more than one shoot during its development. Larvae of $N$. typhae are monophagous on $T$. latifolia, while larvae of $A$. sparganii may also use some other species (Skou, 1991), however, in the studied areas both species were apparently functionally monophagous on $T$. latifolia. At the end of July or at the beginning of August larvae pupate (A. sparganii somewhat earlier than $N$. typhae) inside the shoot or between the leaves of the host plant. The pupal period lasts for about one month in both species.

Two principal species of solitary parasitoids, the koinobiont Spilichneumon limnophilus Thomson and the idiobiont Chasmias paludator Desvignes (Hymenoptera: Ichneumonidae), were found to parasitise larvae and pupae of both $N$. typhae and A. sparganii. A third species parasitising both moths, Vulgichneumon saturatorius L., was rare in most years. These three species were treated as the shared parasitoids in the analyses. The most numerous parasitoid of $N$. typhae, Exephanes occupator Gravenhorst, parasitises $A$. sparganii only occasionally (Teder et al., 1999): in most years no individual $A$. sparganii was parasitised by this species. Other parasitoids treated here as non-shared, accounted for $<1 \%$ of the total parasitism. Like their hosts, studied parasitoids have univoltine life cycles. Adult females overwinter and lay their eggs on moth larvae in late spring / early summer [E. occupator (Hinz \& Horstmann, 2000; pers. obs.) and S. limnophilus (pers. obs.)] or on fresh pupae in July and early August [Ch. paludator (Hinz, 1983; Hinz \& Horstmann, 1999)]. Adult wasps emerge more or less simultaneously with unparasitised moths (E. occupator somewhat earlier). All three parasitoids are oligophagous and are also known to parasitise some other moths (Rasnitsyn \& Siitan, 1981; Hinz \& Horstmann, 1999, 2000) which, however, apparently constitute only a minor fraction of hosts in the habitats studied.

\section{Study areas}

This work was conducted in 7 consecutive years (1995-2001) in southeastern Estonia. Three habitats differing with respect to Typha distribution pattern were examined. The habitat studied in 1995-1996 was characterized by a dense, almost monospecific stand of $T$. latifolia. The vegetation of the habitat examined in 1997-1998 was more heterogeneous with patches of T. lati- folia and Carex elata Bell. ex All. alternating. Both these habitats, with the areas of 3 and 4 ha, respectively, were located in the town of Tartu $\left(58^{\circ} 22^{\prime} \mathrm{N}, 26^{\circ} 45^{\prime} \mathrm{E}\right)$, on the flooded meadow of the Emajõgi River. The third habitat, studied in 1998-2000 was the most heterogeneous where patches of $T$. latifolia (mostly from 0.01 to $0.05 \mathrm{ha}$, at small ponds, ditches, or other damp places) were separated by distances of $0.2-1.0 \mathrm{~km}$. This study area was located in an agricultural landscape, close to the Lake Pangodi (58 $\left.12^{\prime} \mathrm{N}, 26^{\circ} 35^{\prime} \mathrm{E}\right), 20 \mathrm{~km}$ southwest of Tartu. The samples collected from Tartu and Pangodi in 1998 were treated as independent in the analyses.

\section{Sampling}

Field populations were sampled to obtain data on the distribution of moths and the levels of parasitism. Sampling was conducted at the end of July and/or the beginning of August, when moths were in the pupal stage. A variable fraction of $A$. sparganii pupae $(2-51 \%)$ had eclosed by the day of collection. Similarly, a variable, but relatively small fraction of $N$. typhae specimens (4-21\%) were collected as larvae. The fractions of individuals collected before or after the pupal period in a particular sample were regarded as indices reflecting phenology of the species (see below for details). Each year, subsamples from 12-33 plots (173 in total) were collected. In the habitats located in Tartu, subsamples were taken from plots of $2 \times 2 \mathrm{~m}, 3 \times 3 \mathrm{~m}$ or $4 \times 4 \mathrm{~m}$ (depending on larval abundance in the particular year), while in the most heterogeneous habitat (Pangodi) at least 30 individuals ( $N$. typhae $+A$. sparganii) were sampled per each patch. From the study plots, moth pupae were collected by inspecting all shoots carefully. The pupae of both species were relatively large $(2.0-3.5 \mathrm{~cm}$ in length), potential pupation sites were limited, and damaged shoots were easily distinguishable from undamaged shoots. Therefore, it was possible to collect nearly all pupae from the plots. The number of pupae collected in one sample varied from 200 to 2294 (5736 individuals in total; 757 A. sparganii and 4979 N. typhae). Pupae were stored in Petri dishes until eclosion of adult moths or parasitoids. For A. sparganii individuals collected as exuviae, it was always possible to determine whether a moth or a parasitoid, and in most cases, which of the parasitoids, had emerged. The fate (parasitised / unparasitised) of the specimens that died in the laboratory in the early pupal stage (mainly $N$. typhae) could not be determined; they were thus omitted from calculations and analyses ( $5-10 \%$ of specimens each sample).

\section{Data analysis}

Levels of parasitism in $A$. sparganii and $N$. typhae were analysed with respect to relative abundance of the moths and their relative phenology. As the purpose of this study was to examine parasitism-mediated indirect effects, all the calculations and analyses were restricted to plots in which both $A$. sparganii and $N$. typhae were found. Data of such plots were pooled within samples for subsequent analyses. For the same reason, only parasitism caused by shared parasitoids was considered in the analyses. In A. sparganii, shared parasitoids accounted for most of the parasitism. In contrast, for $N$. typhae a considerable fraction of individuals were parasitised by the non-shared $E$. occupator. Although multiple parasitism of hosts previously parasitised by E. occupator was unlikely in this system, these individuals were re-classified as non-parasitised for the analyses. Such an approach was taken assuming that the shared parasitoids, S. limnophilus and Ch. paludator, presumably make their decisions on relative abundance of the two host species on the basis of overall host density (i.e. unparasitised + already parasitised hosts) rather than density of unparasitised hosts. 

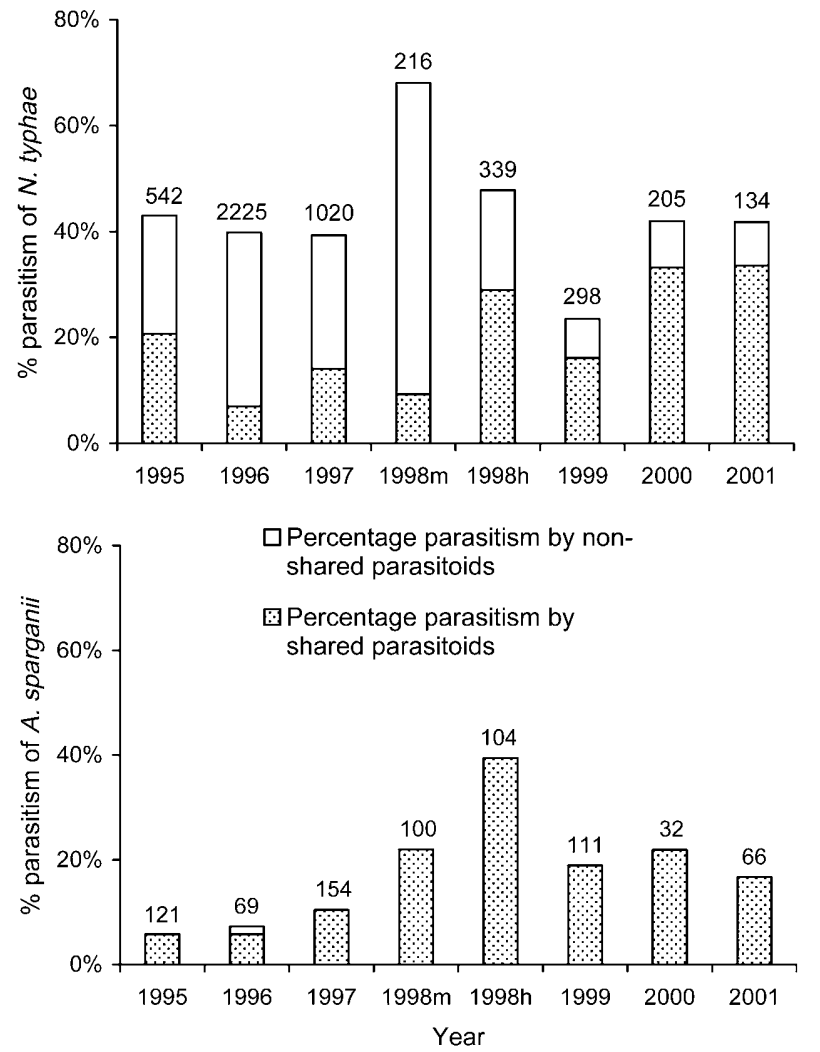

Fig. 1. The levels of shared and total parasitism in A. sparganii and $N$. typhae in the study samples (1998m - sample collected in Tartu, 1998h - sample collected in Pangodi, see text for details). Sample sizes are presented above the bars.

To derive an index describing differences in phenology of the two moths, the study samples were ranked according to 1) the percentage of adults among $A$. sparganii (found as exuviae) on the day of sampling, and 2) the percentage of larvae among $N$. typhae on the day of sampling. The value " 1 " was assigned to the sample of the highest proportion of adults among $A$. sparganii, and to the sample of the lowest proportion of larvae among $N$. typhae, both these ranks indicating the earliest phenologies. Accordingly, the value " 8 " indicated the latest phenologies. The relative phenology index for a particular sample was calculated by subtracting the rank of $A$. sparganii from the corresponding rank of $N$. typhae. The larger was the index obtained in this way, the larger was the difference in phenological distributions of the two moths.

The rationale of this relative phenology index was in describing the degree of temporal overlap, or co-occurrence of the vulnerable stages of the two host species. This may be relevant in the context of indirect interactions. As predicted by the optimal foraging theory, higher abundance of the preferred host should increase parasitoid's selectivity. Accordingly, when the peaks of vulnerable $A$. sparganii and $N$. typhae overlap, more larvae of the preferred host, $N$. typhae, are available, and thus, a larger proportion of larvae of the less preferred species, $A$. sparganii, should escape parasitism. In the opposite case, when the phenological distributions of $A$. sparganii and $N$. typhae differ, a larger proportion of vulnerable $A$. sparganii would fall into the period of lesser choosiness of the parasitoids.

Logistic regression analysis (PROC GENMOD; SAS Institute Inc., 1995) was applied to examine whether the distribution of parasitism between $A$. sparganii and $N$. typhae differs in the study samples. Binomial probability distribution was assumed,

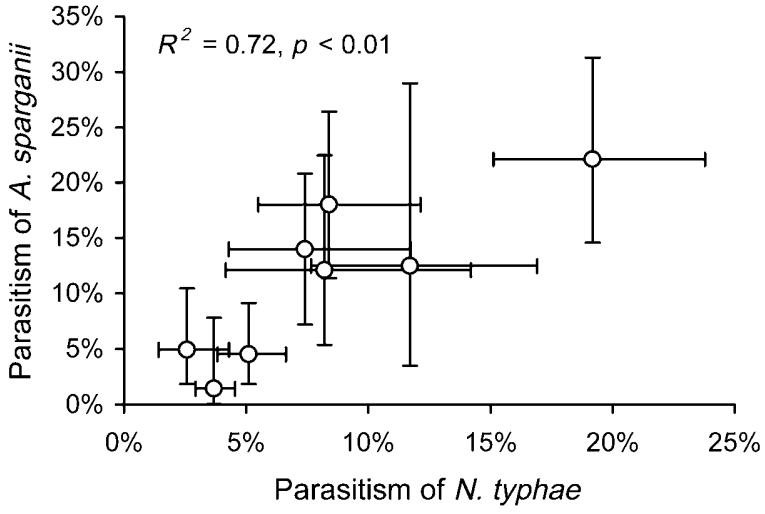

Fig. 2. The relationship between parasitism levels of $A$. sparganii and $N$. typhae. Only parasitism by $S$. limnophilus is considered; $\mathrm{x}$ - and y -error bars represent $95 \%$ confidence intervals for the binomial parameter (parasitised/nonparasitised).

logit was chosen as the link function, DSCALE option was applied to correct for overdispersion. Incidence of parasitism (parasitised/nonparasitised) was used as the response variable, and "sample", "host species" and "sample $\times$ host species" were used as the independent effects. Linear regression was used to examine the effects of relative abundance of the moths and their relative phenological distribution (see above) on their relative distribution of parasitism at the level of sample means. The dependent variable, relative parasitism of the two host species was expressed as the ratio of the fractions of parasitised A. sparganii and $N$. typhae per sample. For example, if in a particular sample, percentage parasitism of $A$. sparganii was $12 \%$ and that of $N$. typhae was $30 \%$, the corresponding index of relative parasitism was $12 / 30=0.4$. The use of this relative measure was chosen to eliminate the effect of overall parasitoid abundance on the parasitism levels of $A$. sparganii. Analogously, relative abundance of the two moths was calculated as the ratio of the numbers of $A$. sparganii and $N$. typhae collected in the same sample.

\section{RESULTS}

Among the samples studied, parasitism levels were highly variable in both of the moth species. Mean values of total percentage parasitism fluctuated from $5.8 \%$ to $38.5 \%$ in $A$. sparganii and from $23.5 \%$ to $68.1 \%$ in N. typhae (Fig. 1). When only shared parasitoids were considered, percentage parasitism of $N$. typhae dropped to the range of $7.0 \%$ to $33.6 \%$, whereas parasitism of $A$. sparganii, being caused mainly by shared parasitoids, changed only negligibly (Fig. 1). Variation in the parasitism levels by individual parasitoid species was also considerable (e.g. parasitism by $S$. limnophilus fluctuated from $2.6 \%$ to $19.2 \%$ in $N$. typhae and from $1.4 \%$ to $22.1 \%$ in $A$. sparganii).

Parasitism levels of the scarcer species, A. sparganii, tended to covary with those of the dominant species, $N$. typhae, the correlation presumably being explained by the overall abundance of the parasitoids. A positive and significant association was observed when parasitism by S. limnophilus was analysed (Fig. 2). For parasitism by $C h$. paludator no association was observed between the parasitism levels of $A$. sparganii and $N$. typhae. 


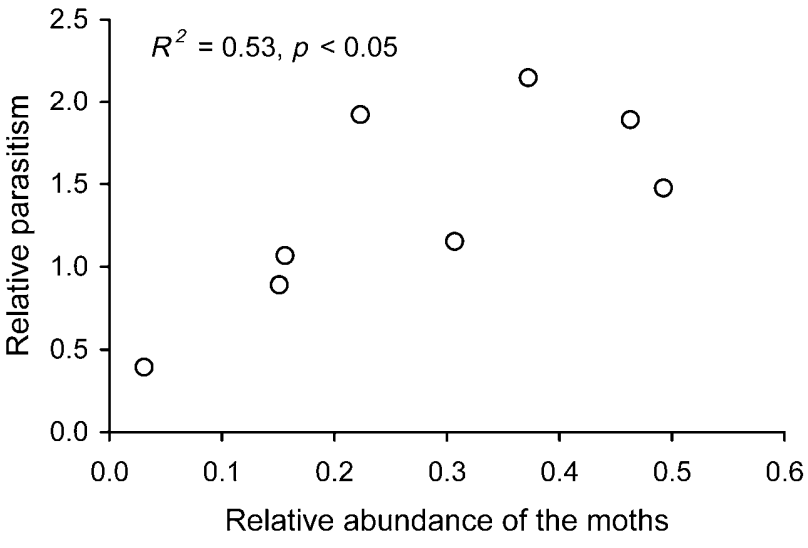

Fig. 3. The relationship between relative abundance of the moths (the ratio of the numbers of $A$. sparganii and $N$. typhae collected in the same sample) and relative parasitism (the ratio of fractions of parasitised $A$. sparganii and $N$. typhae per sample) at the level of means. Only parasitism by S. limnophilus is considered.

The overall levels of parasitism by the shared parasitoids did not differ between the two moth species (Table 1). In contrast to this "overall equality", parasitism levels were not equal in particular study samples. For example, in 1995 parasitism percentage of $N$. typhae by shared parasitoids exceeded that of $A$. sparganii more than 3 times whereas in 1998 (Tartu - site) A. sparganii appeared to suffer from parasitism levels twice higher than $N$. typhae (Fig. 1). The among-sample differences in the relative parasitism of the two host species were statistically confirmed by a highly significant "sample $\times$ host species" interaction (Table 1).

One possible factor able to explain these remarkable differences in relative parasitism levels is the variable relative abundance of the host species. Though $A$. sparganii was less abundant in all samples, relative abundance of $A$. sparganii and $N$. typhae considerably fluctuated among samples. The index of relative abundance of the two species (the number of $A$. sparganii divided by the number of $N$. typhae) fluctuated from 0.03 (1996) to 0.49 (2001). Linear regression of mean values indicated that relative abundance of the moths affected their relative parasitism levels. The effect of relative abundance on relative parasitism levels was positive and significant when parasitism by S. limnophilus was analysed (Fig. 3). Similarly, a positive though not significant trend was observed also when parasitism by $C h$. paludator $\left(R^{2}=0.19, \mathrm{NS}\right)$ was analysed.

TABLE 1. The results of logistic regressions (significance tested by type I analyses) examining the differences in the distribution of parasitism between $A$. sparganii and N. typhae.

\begin{tabular}{lcrc}
\hline Effect & $d f$ & \multicolumn{1}{c}{$\chi^{2}$} & $p$ \\
\hline Incidence of parasitism (0/1) & & & \\
Sample & 7 & 278.8 & $<0.001$ \\
Host species & 1 & 1.8 & 0.19 \\
Sample $\times$ host species & 7 & 39.1 & $<0.001$ \\
\hline
\end{tabular}

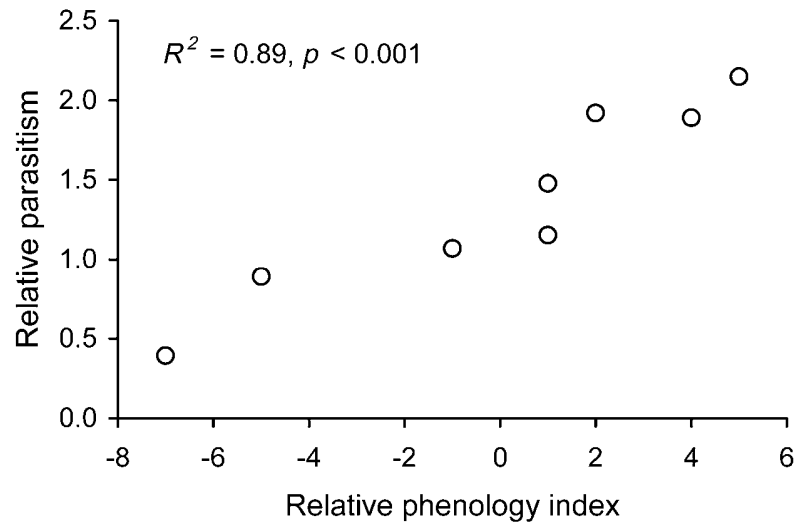

Fig. 4. The relationship between relative phenology index (the larger the index, the larger the difference in phenological distributions of the two species) and relative parasitism (the ratio of the fractions of parasitised $A$. sparganii and $N$. typhae per sample) at the level of means. Only parasitism by $S$. limnophilus is considered.

Another factor that may have differential influence on the availability, and thus, parasitism levels of the two moths, is their relative phenological distribution. Correspondingly, the relative phenology index (see Methods) correlated positively with relative parasitism levels caused by S. limnophilus (Fig. 4). Again, the relationship between relative phenology index and relative parasitism levels by $C h$. paludator $\left(R^{2}=0.11, \mathrm{NS}\right)$ was also positive but non-significant. Mean values of relative abundance and relative phenology index appeared to be correlated $(R=0.81)$. Unfortunately, a two-way ANOVA examining the effects of relative abundance and relative phenology jointly would not have been statistically meaningful due to small sample size ( 8 samples).

\section{DISCUSSION}

The present results revealed a pattern that is consistent with the presence of indirect, parasitoid-mediated interactions between two herbivores. We showed that relative parasitism levels of the two coexisting host species, $A$. sparganii and $N$. typhae, considerably varied among different samples. The present analyses revealed two factors that may contribute to the patterns observed via functional responses of the parasitoids. In particular, relative parasitism levels of the two moths correlated both with relative abundance of the two moths as well as the difference in their phenologies. Although we cannot strictly prove which of the two factors is more important in determination of parasitism levels of $A$. sparganii, the conclusions would be similar: A. sparganii appeared to benefit from the presence of $N$. typhae, or in other words, escape parasitism by its relative scarcity. The case is one of very few (see also Bonsall \& Hassell, 1997; Müller \& Godfray, 1997) providing evidence for indirect interactions in parasitoid-involving systems at a short time-scale (see Holt \& Lawton, 1994). Unfortunately, however, the assymetric distribution of the abundances does not allow us to judge about the symmetry of this interaction (Chaneton \& Bonsall, 2000). 
The effect of relative abundance is likely to be explained by the consequences of frequency-dependent host use by the parasitoids. Such a pattern of host use may result either from the host-frequency-dependent changes in a) host's anti-parasitoid behaviour, or b) foraging behaviour of the parasitoids (i.e. a change in host preference) (for a review, Sherratt \& Harvey, 1993; van Alphen \& Jervis, 1996). The mechanisms of the first type are unlikely in the present system: given the solitary, concealed life-style of the herbivores studied, an increase in the density hardly has a chance to change their defensive behaviour. A change in host preference in parasitoids is a more likely explanation. In the system studied such a switching (sensu Murdoch, 1969) might arise as a consequence of changes in host composition: the higher the $A$. sparganii density, the greater the possibility for parasitoids to learn stimuli deriving from this species, although alternative scenarios are possible (Holt, 1983; Sherratt \& Harvey, 1993).

From the parasitoids' point of view, the relative abundance of a pair of hosts is not necessarily determined by their absolute numbers: host individuals of different ages are rarely equally vulnerable to parasitoid attacks (Briggs \& Latto, 1996; Benrey \& Denno, 1997). The risk of parasitism for the coexisting hosts might thus also depend on the degree of synchrony in hosts' windows of vulnerability. If parasitoids forage for the hosts in a frequencydependent manner, the rarer host may reduce parasitism risk by adjusting its vulnerable stage to occur simultaneously with the more abundant host. The present results are consistent with this scenario: smaller differences in the phenological distributions of the two hosts implied a reduced parasitism risk for $A$. sparganii. Such a pattern may be seen as another indication of a change in host preference in the system studied.

The present system appears to meet the necessary preconditions for switching behaviour to evolve: 1) relative abundance of different prey types should vary either spatially or temporally and 2) predators should be mobile (Cornell, 1976). In the present system, the relative abundance of the two moths varied among samples; shared parasitoids are extremely mobile and can be met even in very isolated patches (Teder \& Tammaru, pers. obs.). Moreover, both moths are quite abundant in absolute terms, they seem to be well suitable for the parasitoids, and their feeding biologies and phenologies are similar. Parasitoids are thus faced with an actual choice of different host species, and switching may appear to be favoured in this system. Such a complex of preconditions is seemingly rarely fulfilled in most natural systems as reflected by rare documentation of changes in host preference in the field (see, however, Kato, 1994; Pike et al., 1999), whereas the phenomenon is repeatedly demonstrated under laboratory conditions (Cornell \& Pimentel, 1978; Chow \& Mackauer, 1991; Drost \& Cardé, 1992).

Frequency-dependent host use has been suggested to have significant consequences for the structure and dynamics of multispecies communities by promoting stability and coexistence of the species (Holt \& Lawton,
1993; Bonsall \& Hassell, 1999; Hassell, 2000). One of the most obvious consequences of parasitism in the studied system is its potential to stabilize population dynamics of the scarcer moth, $A$. sparganii. At lower densities, parasitism levels of $A$. sparganii appeared to be lower which should allow for $A$. sparganii populations to increase. As $N$. typhae populations maintain the density of parasitoids continuously high ( $N$. typhae was the more abundant host species in all samples), the response of parasitoids to an increase in $A$. sparganii density occurs without a delay, a mechanism widely appreciated as promoting stable population dynamics in the host (Holt \& Lawton, 1993). In the studied system, positively densitydependent parasitism accompanying with a change in host preference allows $A$. sparganii to increase when rare thus facilitating coexistence of the host species (see also Holt $\&$ Kotler, 1987). Mechanisms promoting stability for the populations of $N$. typhae remain unclear.

ACKNOWLEDGEMENTS. We thank Heikki Roininen, Robert D. Holt, and two anonymous reviewers for valuable comments, and Rein Karulaas for technical assistance. The study was supported by the grants No. 2147 and 4076 from Estonian Science Foundation.

\section{REFERENCES}

Abrams P.A., Holt R.D. \& Roth J.D. 1998: Apparent competition or apparent mutualism? Shared predation when populations cycle. Ecology 79: 201-212.

ALPHEN VAN J.J.M. \& JERVIS M.A. 1996: Foraging behaviour. In: Jervis M.A. \& Kidd N.A.C. (eds): Insect Natural Enemies: Practical Approaches to Their Study and Evaluation. Chapman \& Hall, London, pp. 1-62.

BenReY B. \& DENNo R.F. 1997: The slow-growth-high-mortality hypothesis: a test using the cabbage butterfly. Ecology 78: 987-999.

Bonsall M.B. \& Hassell M.P. 1997: Apparent competition structures ecological assemblages. Nature 388: 371-373.

Bonsall M.B. \& Hassell M.P. 1999: Parasitoid-mediated effects: apparent competition and the persistence of hostparasitoid assemblages. Res. Popul. Ecol. 41: 59-68.

Briggs C.J. \& LatTo J. 1996: The window of vulnerability and its effect on relative parasitoid abundance. Ecol. Entomol. 21: $128-140$.

Chaneton E.J. \& Bonsall M.B. 2000: Enemy-mediated apparent competition: empirical patterns and the evidence. Oikos 88: 380-394

Chow A. \& Mackauer M. 1991: Patterns of host selection by four species of aphidiid (Hymenoptera) parasitoids: influence of host switching. Ecol. Entomol. 16: 403-410.

Cornell H. 1976: Search strategies and the adaptive significance of switching in some general predators. Am. Nat. 110: 317-320.

Cornell H. \& Pimentel D. 1978: Switching in the parasitoid Nasonia vitripennis and its effects on host competition. Ecology 59: 297-308.

Drost Y.C. \& CARDÉ R.T. 1992: Host switching in Brachymeria intermedia (Hymenoptera: Chalcididae), a pupal endoparasitoid of Lymantria dispar (Lepidoptera: Lymantriidae). Environ. Entomol. 21: 760-766.

Galichet P.F., Cousin M. \& Girard R. 1992: Egg dormancy and synchronization of larval feeding with host plant development in three noctuid (Lepidoptera) species. Acta Oecol. 13: $701-714$. 
Hassell M.P. 2000: The Spatial and Temporal Dynamics of Host-Parasitoid Interactions. Oxford University Press, Oxford, $200 \mathrm{pp}$.

HINZ R. 1983. The biology of the European species of the genus Ichneumon and related species (Hymenoptera: Ichneumonidae). Contrib. Amer. Ent. Inst. 20: 151-152.

Hinz R. \& Horstmann K. 1999: Zur Lebensweise der europäischen Arten von Chasmias Ashmead, 1900 und Limerodops Heinrich, 1949 (Hymenoptera: Ichneumonidae: Ichneumoninae). Entomofauna 20: 301-308.

Hinz R. \& Horstmann K. 2000: Die westpaläarktischen Arten von Exephanes Wesmael. Spixiana 23: 15-32.

Holt R.D. 1983: Optimal foraging and the form of the predator isocline. Am. Nat. 122: 521-541.

Holt R.D. 1987: Prey communities in patchy environments. Oikos 50: 276-290.

Holt R.D. \& Kotler B.P. 1987: Short-term apparent competition. Am. Nat. 130: 412-430.

Holt R.D. \& Lawton J.H. 1993: Apparent competition and enemy-free space in insect host-parasitoid communities. $\mathrm{Am}$. Nat. 142: 623-645.

Holt R.D. \& Lawton J.H. 1994: The ecological consequences of shared natural enemies. Annu. Rev. Ecol. Syst. 25: $495-520$

Houston T.F., Lamont B.B., Radford S. \& Errington S.G. 1993: Apparent mutualism between Verticordia nitens and V. aurea (Myrtacea) and their oil-ingesting bee pollinators (Hymenoptera: Colletidae). Aust. J. Bot. 41: 369-380.

Jeffries M.J. \& Lawton J.H. 1984: Enemy free space and the structure of ecological communities. Biol. J. Linn. Soc. $\mathbf{2 3}$ : 269-286.

Kato M. 1994: Structure, organization, and response of a species-rich parasitoid community to host leafminer population dynamics. Oecologia 97: 17-25.

Müller C.B. \& Godfray H.C.J. 1997: Apparent competition between two aphid species. J. Anim. Ecol. 66: 57-64.

Müller C.B. \& GodfraY H.C.J. 1999: Indirect interactions in aphid-parasitoid communities. Res. Pop. Ecol. 41: 93-106.

MuRdoch W.W. 1969: Switching in general predators: experiments on predator specificity and stability of prey populations. Ecol. Monogr. 39: 335-354.
Olofsson J., Moen J. \& Oksanen L. 1999: On the balance between positive and negative plant interactions in harsh environments. Oikos 86: 539-543.

Pike K.S., Stary P., Miller T., Allison D., Graf G., Boydston L., Miller R. \& Gillespie R. 1999: Host range and habitats of the aphid parasitoid Diaeretiella rapae (Hymenoptera: Aphidiidae) in Washington State. Environ. Entomol. 28: 61-71.

Rasnitsyn A.P. \& SiITAN U.V. 1981: Subfamily Ichneumoninae. In: Medvedev G.S. (ed.): Keys to the Insects of the European Part of the USSR, Vol. 3 (Part 3). Nauka, Leningrad, pp. 506-636. [in Russian]

SAS InstrTUte Inc. 1995: SAS Technical Report P-243. SAS/STAT Software: The GENMOD Procedure, Release 6.09. SAS Institute Inc., Cary, North Carolina, $88 \mathrm{pp}$.

Sherratt T.N. \& Harvey I.F. 1993: Frequency-dependent food selection by arthropods: a review. Biol. J. Linn. Soc. 48: 167-186.

Skou P. 1991: Nordens Ugler. Håndbog over de i Danmark, Norge, Sverige, Finland og Island forekommende arter af Herminiidae og Noctuidae (Lepidoptera), Danmarks Dyreliv 5 [Nordic Owlet Moths. Handbook of Herminiidae and Noctuidae (Lepidoptera) of Denmark, Norway, Sweden, Finland and Iceland. Danish Wildlife 57. Apollo Books, Stenstrup, 566 pp. [in Danish]

Strauss S.Y. 1991: Indirect effects in community ecology: their definition, study and importance. Trends Ecol. Evol. 6: 206-210.

TEDER T. \& TAMmaru T. 2002: Cascading effects of variation in plant vigour on the relative performance of insect herbivores and their parasitoids. Ecol. Entomol. 27: 94-104.

Teder T., Tammaru T. \& Pedmanson R. 1999: Patterns of host use in solitary parasitoids (Hymenoptera, Ichneumonidae): field evidence from a homogenous habitat. Ecography 22: $79-86$.

WоOTTON J.T. 1994: The nature and consequences of indirect effects in ecological communities. Anmu. Rev. Ecol. Syst. 25 $443-466$.

Received July 15, 2002; revised September 19, 2002; accepted September 28, 2002 